\title{
The impact of involvement in mental health research on views about mental health services and service use: Findings from a UK survey
}

\author{
Sue Patterson ${ }^{1 *}$, Jenny Trite ${ }^{2}$ and Tim Weaver ${ }^{3}$ \\ ${ }^{1}$ Principal Research Fellow, Metro North Mental Health, Associate Professor, Applied Psychology, Griffith University Australia, J Floor Mental Health Centre, \\ Royal Brisbane and Women's Hospital, Herston, Queensland 4029, Australia \\ ${ }^{2}$ Independent Service User Researcher, London UK \\ ${ }^{3}$ Associate Professor in Mental Health, Middlesex University, UK
}

\begin{abstract}
Background: Involvement of service users in mental health research, whether predicated on rights-based or instrumentalist arguments, is appropriately understood as a purposive social action likely to have both intended and unintended consequences. Despite rhetoric and some evidence that involvement is a good thing and confers benefits on the people involved, the impact on involved individuals remain underexplored.
\end{abstract}

Aims: To describe the impact of involvement in research on views about mental health services and service use.

Method: A cross-sectional survey of service users involved in mental health research across the UK. Data collected from 166 respondents using an online questionnaire were analysed using the framework approach and narratively synthesised.

Results: Involvement, through enabling 'behind the scenes' access shaped views of service users about services and service use. Increased awareness of challenges and shortcomings of service delivery shifted expectations, commonly empowering service users to proactively engage in treatment. However, for some respondents, disillusionment led to discontinuation of services.

Conclusions: Empowerment is a common outcome of involvement but a minority of service users may become critical and drop out of services. People considering involvement in research should be informed about the potential impact on their views and service use and be appropriately supported during involvement. The theory of user involvement needed to support robust examination of process and outcomes must encompass unintended consequences.

\section{Declaration of interest}

The authors declare they have no financial or other conflicts of interest. The research reported here was conducted with in-kind support of the North London Hub of the Mental Health Research Network. The service user reference group was funded by a grant from the Mental Health Research Network. The work has benefited from discussion with colleagues following a presentation of a summary of findings at the Service User in Academia Conference in Wellington, NZ in December 2014.

\section{Introduction}

The active involvement of people with experience of mental illness and/or service use in mainstream mental health research is predicated variously on ethical, rights-based, and instrumentalist grounds [1] Disentangling these non-exclusive arguments is somewhat arbitrary, but, simply involvement is promoted either as the end goal, valued regardless of any outcomes that might ensue or as a means to a socially valued end. The 'end' most commonly invoked in policy and by advocates of involvement, is the improvement in the quality of research. Although often left unsaid, the corollaries of this are the development of policy- and practice-enabling improvements in outcomes for people who experience mental illness, and hence, public good. The means to the end (which is inherently political in that it necessarily involves with challenging the traditional biomedical control of knowledge related to mental illness) is the complementary incorporation, with specialist researcher 'know-how' (to do research), of expertise grounded in experience of mental distress and service use. Evidence, though patchy [2], suggests that inclusion of such experientially based knowledge can improve the experienced process and outcomes of mental health research [3-6].

Another 'end' is also invoked in arguments for involvement. Although typically subsidiary to arguments for 'the collective good', involvement is also promoted as 'good for' the individuals involved. Notwithstanding some accounts that involvement can be frustrating

Correspondence to: Dr. Sue Patterson, Principal Research Fellow, Metro North Mental Health, Associate Professor, Applied Psychology, Griffith University Australia, J Floor Mental Health Centre, Royal Brisbane and Women's Hospital, Herston, Queensland 4029, Australia, Tel: +61 73646 1153; Fax: +61 73646 1153; E-mail: Susan.patterson@health.qld.gov.au

Key words: user involvement, research, mental health, impact, survey, qualitative Received: August 13, 2016; Accepted: August 29, 2016; Published: August 31, 2016 
and impact negatively on mental health $[7,8]$, the scant research to date generally supports the view that involvement is positive [6]. Involvement has, amongst people with lived experience of mental illness, been associated with various benefits. These include enabling recognition of personal strengths and abilities, greater self-awareness, self-respect, self-esteem, and self-confidence, improved quality of life, and increased social inclusion [8-10].

The findings outlined above and related reviews [11,12] indicate that user involvement in mental health research can, as proposed, influence the quality of research and shape the ways people think and experience themselves and their social worlds [13]. However, as a purposive social action, involvement is also likely to have unintended, consequences that may be negative or regressive [14,15]. With optimizing the practice and outcomes of involvement dependent on understanding impact there is a pressing need to extend the scope of inquiry into impact of involvement generally (Crocker et al. 2016) and on individuals $[12,16]$.

With mental health research, by definition concerned with issues related to mental illness, treatments, and services, it is logical that involvement will potentially influence people's perceptions, experience, and use of services (i.e., those areas in which users have particular and unique experience). However, to our knowledge, and apart from our own brief account (Authors) this potential unintended consequence of involvement remains unexplored. Our aim in expanding previous findings here is two-fold: first to amplify the voice of the service user researchers who, in sharing their insightful accounts showed this to be an important concern and, secondly to extend the conversation about how involvement may affect people involved and assess the implications for enhancing mechanisms for the involvement of service users in research.

\section{Method}

As part of a broader study into the roles, activity, and experience of service users involved in mental health research in the UK (Authors), we set out to describe the impact of involvement in research on service users' views about mental health services and use of mental health services.

We analysed data from a cross-sectional survey of people with experience of mental health service use, involved in mental health research in the UK, conducted in 2011-2012. As described fully elsewhere, the survey employed a snowball strategy to disseminate a URL link to an online questionnaire embedded in an invitation email through universities, charities, and research and service user networks. Recipients of the email were asked to consider their own eligibility (i.e., personal experience of mental illness/mental health service use, and involvement in mental health research as other than a participant), complete the questionnaire if eligible, and forward the invitation through personal and professional networks. The questionnaire (available on request) comprised multiple-choice items with which participants were invited to rate strength of agreement/disagreement and open questions, one of which was ' How has involvement in research influenced your use of, or attitude to $\mathrm{MH}$ [mental health] services?' Questionnaire completion was anonymous. Ethical approval for the survey was obtained from Fulham Research Ethics Committee $(11 / \mathrm{LO} / 1480)$.

\section{Analysis}

Data were downloaded from Survey Monkey website to SPSSv20
(IBM Corp, 2011) and Microsoft applications for analysis. Descriptive statistics were used to profile the sample and quantify involvement activity. Responses to open questions were subject to analysis using the framework approach [17], selected because it employs both deductive and inductive logic to answer pre-determined questions and discern subtleties in data. The first step in analysis was to populate an initial frame comprising cells representing research question and 'respondent', with data from open questions, with multiple allocations possible. A constant comparative process was then employed to identify patterns and exceptions in the data, with the analytic frame iteratively developed as themes and categories were developed in the data. Finally, diagramming was used to link key themes in an explanatory frame used to construct the narrative account below. To ensure findings remained true to respondents' accounts, the authors and members of a service user reference group used a dialogic collaborative process [18] to critically review data allocation and analysis in round-table discussions.

\section{Results}

The 166 survey respondents who typically reported extensive service use histories were aged $21-73$ years (median $=47)$, predominately female $(\mathrm{N}=103,63 \%)$, and White British $(\mathrm{N}=127,78 \%)$. The majority $(\mathrm{N}=106$, $63.9 \%)$ completed tertiary education, with seventeen (10\%) holding higher research degrees (i.e., $\mathrm{PhD}$ and $\mathrm{MD}$ ). The sample included people identifying as service users involved in research, and researchers with experience of service use. As detailed elsewhere (Authors), diverse role/position descriptors were used; many respondents identified 'labelling' as a vexed issue with personal and professional implications.

Reported employment status varied, generally, and vis-àvis research. Nearly half $(\mathrm{N}=81)$ were self- or other- employed as researchers, with a similar proportion $(\mathrm{N}=73 ; 44 \%)$ reporting voluntary involvement in mental health research. With median involvement of 5.8 years (range 0.8 to 40 years), respondents were typically undertaking multiple research activities (e.g. data collection, analysis, governance, write up), often in several settings (e.g. health services, universities, charity sector).

Data pertinent to the question 'how has involvement in research influenced your use of, or attitude to, $\mathrm{MH}$ services?" were drawn from all respondents. Three quarters $(\mathrm{N}=125)$ completed the question directly, typically providing a paragraph detailing the ways research involvement had influenced their views about, and use of services.

Speaking to so many different individuals in the course of the work has altered my thoughts and perception of both individual(service users, staff, researchers, academics and the system. (R91: F, 58years; service user researcher, University) A small minority of respondents $(\mathrm{N}=10)$ reported that involvement had no impact on their attitude toward, or use of, services, although, as the following excerpt illustrates, the original views may have been critical.

My attitude hasn't really changed: current service provision is generally woefully under-funded and in many cases, the quality of services is lottery-like. (R130: M, 30years; service user researcher, NHS)

Most commonly, respondents wrote of increasing knowledge and understanding of the complexity of mental health service delivery.

Being a service user researcher has opened my eyes to the other side in terms of learning more about systems in mental health trusts from a staff/researcher perspective. (R147: F, 46years; self-employed service user researcher)

Being part of research teams, engaging in various ways with service 
providers and other service users while collecting and analysing data, and undertaking literature reviews had supported insights regarding the entangled socio-political and structural influences on service delivery.

Yes, my attitude has changed. The value I place on mental health services has, if anything, increased. However, at the same time, I am hugely concerned at the lack of evidence, co-ordination, standards, quality, communication and protectionism, which appear to dominate the area. (R43: M, 39 years; self-employed service user researcher) Respondents described both increased appreciation of the constraints on services and identification of deficiencies in management and practice. Being actively involved I have come to see how the mental health services lag far behind in implementation of best practice. I fight shy and am very careful of service use in ways that I never would have been before. (R96: M, 60 years; volunteer, various settings) Referencing the political nature of madness, pervasive stigma, burden of bureaucracy, financial constraints, and dominance of the medical model, respondents noted a lack of congruence between policy and rhetoric around 'recovery' and practice. With increasing knowledge about mental illness and the various interventions research had shown to be useful, respondents were critical of the variable access to interventions and heavy reliance on medication they observed in services.

I am much more critical of the lack of evidence behind many of the treatments and services offered. (R137: M, 51years; self-employed researcher) My experience has made me feel increasingly that mental health services in their present form conflict with how I account for my personal experience ... I am more cynical about the ability of statutory services to support me effectively. (R42: F, 37 years; service user researcher, University) Research involvement had drawn respondents' attention to the heterogeneity of service users and their diverse needs and expectations of services. Developing 'different' relationships with service providers had provided insights regarding the pressures faced by managers and clinicians who were generally, but not universally, considered well intentioned.

The majority of respondents also reported that their enhanced understanding of the big picture and challenges faced by services gave rise to new-found respect for service providers. Shifted relationship with mental health professionals - less suspicious of them! I think I am more willing to look for support from mental health services now than I used to be. (R88: F, 57 years; researcher, NHS).

These views were concordant with those of the several respondents who reported becoming more 'realistic' or 'pragmatic' in expectations of services. Notwithstanding modification of expectations, respondents also noted that, rather than justify apparent service inertia and maintenance of the status quo, the complexities of service structures and diversity of needs obliged improvement.

I can see two sides of a situation, the drive to roll out services that tick all the boxes in terms of funding and staff, but also see the absolute need for individuals to be treated as such, with their individual needs needing to be considered first and foremost. (R67: F47years; researcher, Charity) I have found that my patience for sloppy or damaging practice with regards to the treatment of my own mental health is getting less and less the more involved in research I become. (R30: F, 28 years; researcher, Charity) For a minority, involvement in research had affirmed or supported development of a critical position. Three respondents wrote of increasing disdain for services and 'opting out'. [research involvement] has made stay as far away from them as possible, I will not get involved with secondary services. (R116: F, 51 years; self-employed 'Survivor Researcher') I am increasingly being pulled towards the arguments of the Critical Psychiatry and Alternatives to Psychiatry movement. (R42: F, 37 years; researcher, University) More commonly, however, observation of the need for improvement validated and enhanced commitment to working with services to achieve the desired changes.

My attitude to mental health services is critical. However I am aware that psychiatry and its practices cannot be ignored by serviceusers who want to bring new ideas (and sometimes old ideas) into the consulting room; we will have to work together. That is our only chance of engaging sufficiently with clinicians and researchers to change their minds sufficiently to make 'nothing about us, without us' a reality. (R134: M, 44years; researcher, University).

It has made me more determined to be part of a movement that tries to redefine what works for us and improve provision. It reinforces how blunt an instrument psychiatry is! (R112: F, 47 years; self-employed 'Survivor Researcher').

\section{Empowerment and active engagement in treatment}

The most commonly reported impact of involvement in research on respondents' service use was adoption of a proactive, critical engagement in care. Twenty-two respondents described themselves specifically as 'empowered' in relation to services and service providers. Many others referred to feeling in control, increased confidence interacting with health professionals, and/or realisation that professionals were not omnipotent. Armed with knowledge and understanding of their own conditions, treatment options, and the ways services 'should' work, developed through research activities, respondents described being more assertive during consultations. They described questioning professional advice, and emergent openness to considering alternate treatments, leading to better service and outcomes. my competence in research has empowered me to take an active role in my treatment instead of passively doing whatever they tell me. (R2: F, 25 years; PhD student).

I have learned so much. Enough to finally stand up to my psychiatrist and challenge the way he was, and wanted to treat me. I managed to come up with my own theories about why I, and perhaps others with similar diagnoses, are the way we are. I used to believe psychiatry that the only thing between me and psychosis was the medication (and that was right - then) but now I have so, so many more ways of coping. (R134: M, 44 years; researcher, University).

I am more informed about available treatments... I'm less inclined to use medication on an ongoing basis. I'm less inclined to trust psychiatrists. I'm more inclined to take responsibility for my health instead. (R109: M, age not given, volunteer, NHS trust) Taking more responsibility for their health, respondents were more likely to question the views of professionals and construe them as a resource or partner rather than expert.

I used self-help and mindfulness techniques that I conducted research into to overcome a brief episode of depression... Had I been in a similar situation without this knowledge, I am sure I would have experienced another prolonged episode. (R137: M, 51 years; selfemployed researcher/consultant).

Through work I have realised my mental health is to a large extent my responsibility whereas before I thought the system should/could make me better. Through collecting data from others I have heard 
many sides of many stories- this has informed my thinking. (R91: F, 58years; service user researcher, University) A minority, however, having come to view the system and services as intransigent, were less hopeful of influencing their own care. Even the most influential user/survivor researcher in mental health will probably not be able to control interventions used on them in the system. (R132: F, 40years; service user researcher.)

\section{Discussion}

Analysis of data collected anonymously from 166 service users actively engaged in mental health research has shown that involvement often has a substantial impact on views about services and service use. Involvement, through enabling access to information and development of insight to the complexities of mental health service delivery, commonly led to critical consideration of disjunction between mental health policy and practice in services. Involvement with research led to identification of gaps and inconsistency in service provision and concerns about the treatments provided (or not provided). Engagement, as other than a 'patient', with research teams, service providers, and service user peers supported appreciation of the difficulties of service delivery, leading to more 'realistic' or 'pragmatic' expectations of services. Involvement also supported personal development (e.g. increased confidence and assertiveness). Coupled with newly developed knowledge about evidence based treatments and their rights as service users, this promoted a more proactive approach to service use. Involved service users typically felt empowered to challenge the status quo, to seek second opinions and to take responsibility for their own mental health care. These findings are consistent with research linking involvement with empowerment, re-balancing of professional/ patient relationships, and development of capacity to speak up in other contexts [11], for example, with Aboriginal Women [19], forensic patients [20] and people with arthritis [21]. The predominance of such 'positive' outcomes should not overshadow our finding that involvement, for an important minority of respondents, had led to, or entrenched, a critical perspective and views about services, and for some, discontinuation of service use.

Before considering implications of our findings we note some study limitations, which constrain interpretation. The principal limitation relates to the (unknowable) representativeness of the sample and experiences reported. In the absence of a census of service users involved in research in the UK, we employed a snowball recruitment strategy to optimise reach, inviting potential participants to assess their own eligibility against broad criteria: experience of mental illness, use of mental health services, and involvement in mental health research as other than a participant. It is possible that the invitation did not reach all potential participants and that the experiences of those who responded differ from those who did not. We note too, that the cross-sectional data represent respondents' constructions of events at a particular time; further experiences or time may develop, modify, or change these. Moreover, findings may be vulnerable to claims of subjectivity inherent in qualitative research [22]. In mitigation of these limitations related to study design, we note that the heterogeneity of respondents and advice from academic and service user networks that the invitation to participate circulated widely and repeatedly, encourage confidence that the data represent a diverse sample. Importantly, respondents were able to complete the survey in their own time anonymously, reducing potential influences on their account of experiences. Our systematic analysis was guided by a group of service user researchers, and we ground our findings in data. We offer a credible account, which extends critical discussion of the impact of service user involvement in mental health research. In moving beyond impact on the research agenda and process and outcomes of research, findings have implications for the theory and practice of user involvement.

As outlined above, user involvement in research may be understood as an ethical mandate or democratic right, consistent with Lincoln's maxim 'of the people, by the people, for the people', or the mantra of the disability movement 'nothing about us without us'. Involvement is also supported as a means to improving outcomes for Patients and The Public, and individuals who are involved [23]. However, beyond general references to improving the quality of research process and outcomes and personal development, mechanisms by which goals are to be achieved are seldom explicit and just, which mechanisms of involvement might optimise impact, remains uncertain [12]. User involvement in research it has been argued has been underconceptualised; thus, theoretical development is required [11,12]. Our findings can contribute to the much needed theory of involvement, and have implications for practice.

Regarding theory development, involvement in mental health research influenced service users' views about services and, consequently, use of services (in various ways) contributed new information to the logic of the 'how' user involvement, which might contribute to change consistent with social good. Whether survey respondents reported becoming more proactive in engagement with services or 'opting out' of formal treatment, involvement was commonly associated with personal empowerment. It may be that service users whose perspectives shift through involvement and elect to remain engaged, challenging the status quo will influence the practice of the clinicians with whom they work. This may be a mechanism that supports change in the balance of power within mental health services, which is central to the development of person-centred services promoted internationally. This unintended and distal impact requires further investigation.

The key implication of our findings for practice of involvement is the importance of acknowledging the potential for involvement to affect views about services and service use. It seems appropriate that, just as voluntary participation in research is predicated on consent based in understanding of the possible risks and benefits, active involvement in the research process should be similarly informed. We contend that service users considering involvement in research have a right to know that exposure to parts of mental health and research systems to which they may not otherwise have access may lead to shifting perceptions. This may have been positive in terms of the appreciation and greater understanding of the political, financial, and organisational context in which services operated, but its product was often disillusionment, and it led some to disengagement from services.

While the "ultimate aim of involvement is to have outsiders influence research" (Oliver et al., 2015), following Merton', we conclude that involvement of service users in mainstream research will necessarily impact the lives of people involved in ways which may not be intended. Involvement necessarily shapes views, actions, and experiences of those involved, in ways that we just now begin to explore systematically. There is a pressing need for further research to explore the impact of involvement on individuals and that this should be at the heart of development of a conceptual understanding, which is critical to optimising practice and the much needed research into impact [2] enabling assessment of return on investment. 


\section{References}

1. Beresford P (2002) User involvement in research and evaluation: Liberation or regulation. Social Policy and Society 1: 95-105.

2. Crocker J, Boylan AM, Bostock MA, Locock L (2016) Is it worth it? Patient and public views on the impact of their involvement in health research and its assessment: a UKbased qualitative interview study. Health Expectations.

3. Ennis L, Wykes T (2013) Impact of patient involvement in mental health research: longitudinal study. Br J Psychiatry 203: 381-386. [Crossref]

4. Gillard S, Simons L, Turner K, Lucock M, Edwards C, et al. (2012) Patient and public involvement in the coproduction of knowledge: Reflection on the analysis of qualitative data in a mental health study. Qualitative Health Research: 1126-1137.

5. Rose D, Leese M, Oliver D, Sidhu R, Bennewith O, et al. (2011) A comparison of participant information elicited by service user and non-service user researchers. Psychiatric Services 6: 210-213.

6. Staley K (2009) Exploring Impact: Public involvement in NHS, public health and social care research. Eastleigh, STATE: INVOLVE.

7. Clark M, Glasby J, Lester H (2004) Cases for change: User involvement in mental health services and research. Research Policy and Planning: 31-38.

8. Patterson S, Trite J, Weaver T (2014) Activity and views of service users involved in mental health research: UK survey. Br J Psychiatry 205: 68-75. [Crossref]

9. Mental Health Foundation (2003). Surviving user-led research: Reflections on supporting user-led research projects. London: Mental Health Foundation.

10. Sayce L, Morris D (1999) Outsiders coming in? Achieving social inclusion for people with mental health problems. London: Mind Publications.

11. Brett J, Staniszewska S, Mockford C, Herron-Marx S, Hughes J, et al. (2014) Mapping the impact of patient and public involvement on health and social care research: a systematic review. Health Expect 17: 637-650. [Crossref]
12. Domecq JP, Prutsky G, Elraiyah T, Wang Z, Nabhan M, et al. (2014) Patient engagement in research: a systematic review. BMC Health Serv Res 14: 89. [Crossref]

13. Oliver S, Liabo K, Stewart R, Rees R4 (2015) Public involvement in research: making sense of the diversity. J Health Serv Res Policy 20: 45-51. [Crossref]

14. Beresford P (2003) User involvement in research: exploring the challenges. Nursing Times Research 8: 36-46.

15. Merton RK (1936) The unanticipated consequences of purposive social action American Sociological Review: 895.

16. Mockford C, Staniszewska S, Griffiths F, Herron-Marx S (2012) The impact of patient and public involvement on UK NHS health care: a systematic review. Int J Qual Health Care 24: 28-38. [Crossref]

17. Gale NK, Heath G, Cameron E, Rashid S, Redwood S, et al. (2013). Using the framework method for the analysis of qualitative data in multi-disciplinary health research. BMC Medical Research Methodology 1: 117.

18. Patterson SE, Hart J, Weaver TD (2010) Delusions and qualitative confusions: a dialogic collaborative exploration. Qual Health Res 20: 1008-1018. [Crossref]

19. Dickson G, Green K (2001) Participatory action research: Lessons learned with Aboriginal grandmothers. Health Care for Women International: 471-482.

20. Beer D, Keeble P, MacInnes D, Rees D, Reid L, et al. (2005) Development of a questionnaire to measure service user satisfaction within inpatient forensic services - The Forensic Satisfaction Scale. National Programme on mForensic Mental Health R\&D, Liverpool.

21. Hewlett S, Wit Md, Richards P, Quest E, Hughes R, et al. (2006) Patients and professionals as research partners: challenges, practicalities, and benefits. Arthritis Rheum 55: 676-680. [Crossref]

22. Sullivan GB (2002) Reflexivity and subjectivity in qualitative research: The utility of a Wittgensteinian framework. Forum: Qualitative Social Research 3.

23. National Institute for Health Research (INVOLVE project). Equity and excellence: liberating the NHS. NIHR.

Copyright: (C2016 Patterson S. This is an open-access article distributed under the terms of the Creative Commons Attribution License, which permits unrestricted use, distribution, and reproduction in any medium, provided the original author and source are credited. 